\title{
Electronic topological transitions and mechanical properties of hafnium dioxide allotrope at high pressure: Evolutionary first-principles techniques
}

Thipok Bovornratanaraks

Triam Udom Suksa School

Rajeev Ahuja

Indian Institute of Technology (IIT) Ropar

Prutthipong Tsuppayakorn-aek ( $\sim$ Prutthipong.Tsup@gmail.com )

Chulalongkorn University

\section{Research Article}

Keywords: Hafnium dioxide, structural prediction, semiconductor, mechanical properties, high pressure

Posted Date: September 21st, 2021

DOl: https://doi.org/10.21203/rs.3.rs-907707/v1

License: (c) (i) This work is licensed under a Creative Commons Attribution 4.0 International License.

Read Full License 


\title{
Electronic topological transitions and mechanical properties of hafnium dioxide allotrope at high pressure: Evolutionary first-principles techniques
}

\author{
Thipok Bovornratanaraks ${ }^{\mathrm{a}, \mathrm{b}}$, Rajeev Ahuja ${ }^{\mathrm{c}, \mathrm{d}}$, Prutthipong \\ Tsuppayakorn-aek ${ }^{\mathrm{e}, \mathrm{f}}$ \\ ${ }^{a}$ Triam Udom Suksa School 227 Phyathai Road Patumwan Bangkok 10330 Thailand \\ ${ }^{b}$ Development and Promotion of Science and Technology Talents Project (DPST) The \\ Institute for the Promotion of Teaching Science and Technology (IPST) 924 Sukhumvit \\ Rd Phra Khanong Khlong Toei Bangkok 10110 \\ ${ }^{c}$ Condensed Matter Theory Group Department of Physics and Materials Science Uppsala \\ University Box 530 SE-751 21 Uppsala Sweden \\ ${ }^{d}$ Department of Physics Indian Institute of Technology (IIT) Ropar Rupnagar 140001 \\ Punjab India \\ ${ }^{e}$ Extreme Conditions Physics Research Laboratory (ECPRL) and Physics of Energy \\ Materials Research Unit Department of Physics Faculty of Science Chulalongkorn \\ University 10330 Thailand \\ ${ }^{f}$ Thailand Center of Excellence in Physics Ministry of Higher Education Science \\ Research and Innovation 328 Si Ayutthaya Road Bangkok 10400 Thailand
}

\begin{abstract}
Allotrope of $\mathrm{HfO}_{2}$ is explored by using first-principles evolutionary algorithm technique, based on density functional theory. The tetragonal structure with a space group of $\mathrm{P} 4 / \mathrm{nmm}$ is found to be themodynamically stable within the harmonic level. Arising particularly from the relative enthalpy, hafnium dioxide allotrope is taken into account in appraising the dynamic stability. Following this, the phonon calculations display that hafnium dioxide allotrope is dynamically stable under compressed conditions. Along with, the density of states suggests that hafnium dioxide allotrope is demonstrated that it is semiconductor. Besides, the more significant change in the shape of density of states is observed when the pressure increased, by adopting an effect of this electronic topological transition, resulting in the energy gap is falling down monotonically. By inspecting their elastic constants and Vicker's
\end{abstract}

Email address: prutthipong.tsup@gmail.com (Prutthipong Tsuppayakorn-aek) 
hardness, the P4/nmm structure displayed the Vicker's hardness of $26.1 \mathrm{GPa}$ at a pressure of $200 \mathrm{GPa}$. These findings suggest $\mathrm{HfO}_{2}$ is more likely to be attained experimentally and theoretically in metal oxides family.

Keywords: Hafnium dioxide, structural prediction, semiconductor, mechanical properties, high pressure

PACS: 0000, 1111

2000 MSC: 0000, 1111

\section{Introduction}

An enhancement of superhard materials aims to go beyond diamond, particulary diamond is the superhard material and is the hardest $[1,2,3,4]$, with a harness value of $96 \mathrm{GPa}$ by the experimental observation. [2] This fact is well known in diamond is transformed from graphite under compression at a pressure of $15 \mathrm{GPa}$. [5] In addition to this, it was pointed out that diamond is the crystallography of carbon, and carbon is provided in low- $Z$ elements. [3] Along with, low- $Z$ elements related to low- $Z$ compounds such $\mathrm{B}_{4} \mathrm{C}, \mathrm{C}-\mathrm{N}$, $\mathrm{SiC}$, and $\mathrm{SiO}_{2}$. At this time, metal oxide is one of the series of materials with hardness materials - for example, $\mathrm{SiO}_{2}, \mathrm{RuO}_{2}, \mathrm{AlO}_{2}, \mathrm{YO}_{2}$, and $\mathrm{ZrO}_{2}$, respectively.[6]

As already investigated by Chen et al., [6] it is interesting to note transition metal oxides (TMOs), i.e., $\mathrm{RuO}_{2}, \mathrm{YO}_{2}$, and $\mathrm{ZrO}_{2}$, respectively. It should be noted that $\mathrm{RuO}_{2}$ is theoretically reported the Vicker's hardness of 23 GPa. Recently, Mazumder et al., [7] also theoretically calculated Vicker's hardness of $\mathrm{TiO}_{2}, \mathrm{SnO}_{2}, \mathrm{ZrO}_{2}$ and $\mathrm{HfO}_{2}$, respectively. Following this, it was found that $\mathrm{HfO}_{2}$ exhibited Vicker's hardness of $11.59 \mathrm{GPa}$ : it has higher than $\mathrm{TiO}_{2}, \mathrm{SnO}_{2}$, and $\mathrm{ZrO}_{2}$. As a result of $\mathrm{HfO}_{2}$, it is should mentioned that $\mathrm{HfO}_{2}$ is notable for hardness property, but also structural behaviour. [7] Also, $\mathrm{HfO}_{2}$ is considered semiconductor with an indirect band gap, resulting in the band gap of $4.62 \mathrm{eV}$. This is, therefore, $\mathrm{HfO}_{2}$ is anticipated to apply as metal-oxide-semiconductor devices in the upcoming generations.

Regarding structural behavior, $\mathrm{HfO}_{2}$ has been particularly studied under compressed conditions. Without a doubt, $\mathrm{HfO}_{2}$ has been observed experimentally by the Raman spectra and x-ray-diffraction technique. [8] As a consequence, the ambient phase, which is a monoclinic structure with the space group $\mathrm{P} 22_{1} / \mathrm{c}$, transformed into an orthorhombic I with the space group $\mathrm{Pbca}$ at a pressure of $10.2 \mathrm{GPa}$. With increasing pressure, it transformed into a or- 
thorhombic II with the space group Pnma at a pressure of $37 \mathrm{GPa}$. Recently, the crystallography of $\mathrm{HfO}_{2}$ has been investigated under high pressure.[9] As a result of this, the Pnma structure is demonstrated that it is a meta-stable because the Pnma structure is dynamically stable, and it is thermodynamically unstable. Following this, the Pnma structure is explored the electronic properties, indicating that it is semiconductivity with the band gap of $3.36, \mathrm{eV}$.

Recently, extensive study of $\mathrm{HfO}_{2}$ was investigated mechanical properties by the experimental observation. [10] The work has been shown that the $\mathrm{HfO}_{2}$ exhibited a significant increase in hardness at least up to $14.4 \mathrm{GPa}$. It is, however, worth to noting that no successful report and attempt to enhance the hardness property of $\mathrm{HfO}_{2}$ at high pressure. Additionally, the Pnma structure has been reported by Zhang et.al.[9], it is the meta-stable structure. Also, one might think of the Pnma structure is possible to be a high-temperature phase, if consider an effect of entropy. For this reason, to the best of our knowledge, it might be worth trying to think of allotrope of $\mathrm{HfO}_{2}$ because it is possible to existence of another meta-stable phase at a temperature of $0 \mathrm{~K}$.

Thus, in this work, $\mathrm{HfO}_{2}$ is explored by using an evolutionary techniques, based on density functional theory. One of the well-known the Universal Structure Predictor: Evolutionary Xtallography (USPEX). [11], it will be quite intuitive to give an insight into the possible allotope of $\mathrm{HfO}_{2}$. Arising particularly from the relative enthalpy, hafnium dioxide allotrope is taken into account in appraising the stable structure at a temperature of $0 \mathrm{~K}$. Following this, the phonon calculations display that hafnium dioxide allotrope is dynamically stable under compressed conditions. By considering the electronic properties, hafnium dioxide allotrope is demonstrated that it is semiconductor. Regarding hardness properties, we used the Chen model [6] to determine the Vicker harness. Interestingly, these Vicker's harness values are rather high in comparison with those estimated for $\mathrm{RuO}_{2}$.

\section{Computational details}

The searching for an allotrope of hafnium oxide compound was performed by first-principles evolutionary techniques, as implemented the Universal Structure Predictor: Evolutionary Xtallography (USPEX). [11] and the Vienna ab initio simulation package (VASP) [12] which utilizes the density functional theory. In all subsequent generations, the random symmetric al- 
gorithm employed $40 \%$ heredity, $20 \%$ random symmetric, $20 \%$ soft mutation, and $20 \%$ transmutation operators in the pressure range from 150 to $200 \mathrm{GPa}$ with structures containing up to four formula units. All structures were fully relaxed using the generalized gradient approximation of the PerdewBurke-Ernzerhof (GGA-PBE) functional [13] for the exchange-correlation functional. We employed the projector augmented wave (PAW) method [14] and the conjugate gradient scheme, as implemented in the VASP [12]. The pseudocore radii of $\mathrm{Hf}$ and $\mathrm{O}$ are $2.4 \mathrm{Bohr}$ and $1.1 \mathrm{Bohr}$, respectively, which are small enough to ensure that no overlap of spheres will occur under extreme condition. The tetragonal structure was calculated with an initial Brillouinzone (BZ) sampling grid of spacing $2 \pi \times 0.02 \AA^{-1}$ in order to guarantee the convergence of the derived ground-state energy. To confirm the dynamical stability, the structure was calculated by using the ab initio lattice dynamics with the supercell approach, as implemented in the VASP code together with the PHONOPY package [15].

\section{Results and discussion}

As aforementioned in the section of introduction and computational details, we introduce here a allotrope of $\mathrm{HfO}_{2}$ with high-pressure phase is predicted at high pressure. In present work, our study opens the door to the exploration of the allotrope of $\mathrm{HfO}_{2}$ above $150 \mathrm{GPa}$. We have used USPEX to predict the $\mathrm{HfO}_{2}$ structure under high pressure; the result gave two particularly low-enthalpy structures, indicating that they are a orthorhombic structure and a tetragonal structure with the space group of Pnma and the space group of $\mathrm{P} 4 / \mathrm{nmm}$, as shown in Figure 1. Also, the structural details is shown in Table- 1 . As a result of this, the structures calculated the relative enthalpy by considering the convex hulls, as shown in Figure 2. Following this, we demonstrated the thermodynamic stability from 150 to $200 \mathrm{GPa}$ by using first-principles calculations, based on density functional theory with the PBE formalism of GGA. At this point, it should be mentioned that our calculations were performed at a temperature of $0 \mathrm{~K}$, indicating that the enthalpy can confirm a phase stability under high pressure. $[16,17,18,19,20,21,22,23]$ This is due to the fact that there is no entropy contribution. Also, the relationship between pure elements $\mathrm{Hf}$ and $\mathrm{O}$ indicated the the relative enthalpy of $\mathrm{HfO}_{2}$. We considered the compositions of $\mathrm{HfO}_{2}$, bcc-Hf [24] and $\mathrm{C} 2 / \mathrm{m}-\mathrm{O}$. [25] The stability of $\mathrm{HfO}_{2}$ is presented within the connected lower convex wrapper, indicating that there is a can- 
Table 1: Structures of the $\mathrm{HfO}_{2}$ at $150 \mathrm{GPa}$.

\begin{tabular}{lll}
\hline Structure & Lattice parameters $\left(\AA,^{\circ}\right)$ & Atomic coordinates \\
\hline Pnma & $\mathrm{a}=4.9309 \mathrm{~b}=2.9261 \mathrm{c}=5.9259$ & Hf $(0.747,0.250,0.123)$ \\
& $\alpha=90 \beta=90 \gamma=90$ & $\mathrm{O}(0.355,0.250,0.677)$ \\
$\mathrm{P} 4 / \mathrm{nmm}$ & $\mathrm{a}=2.9518 \mathrm{~b}=2.9518 \mathrm{c}=5.0428$ & $\mathrm{Hf}(0.966,0.250,0.826)$ \\
& $\alpha=90 \beta=90 \gamma=90$ & $\mathrm{O}(0.000,0.500,0.862)$ \\
& & $\mathrm{O}(0.000,0.000,0.500)$ \\
\hline
\end{tabular}

didate high-pressure phase found above $150 \mathrm{GPa}$ : the tetragonal structure with the space group of $\mathrm{P} 4 / \mathrm{nmm}$. Considering the pressure of $150 \mathrm{GPa}$, the aforementioned computational findings manifested that the Pnma structure is a meta-stable structure, and it is in good agreement with those previously reported by Zhang et.al.[9]. In fact, our calculations carried out at a temperature of $0 \mathrm{~K}$ and this is, however, beyond the scope of this work for consideration at high temperature. We thus propose that the issue clearly deserves further investigation by considering the effect of entropy.
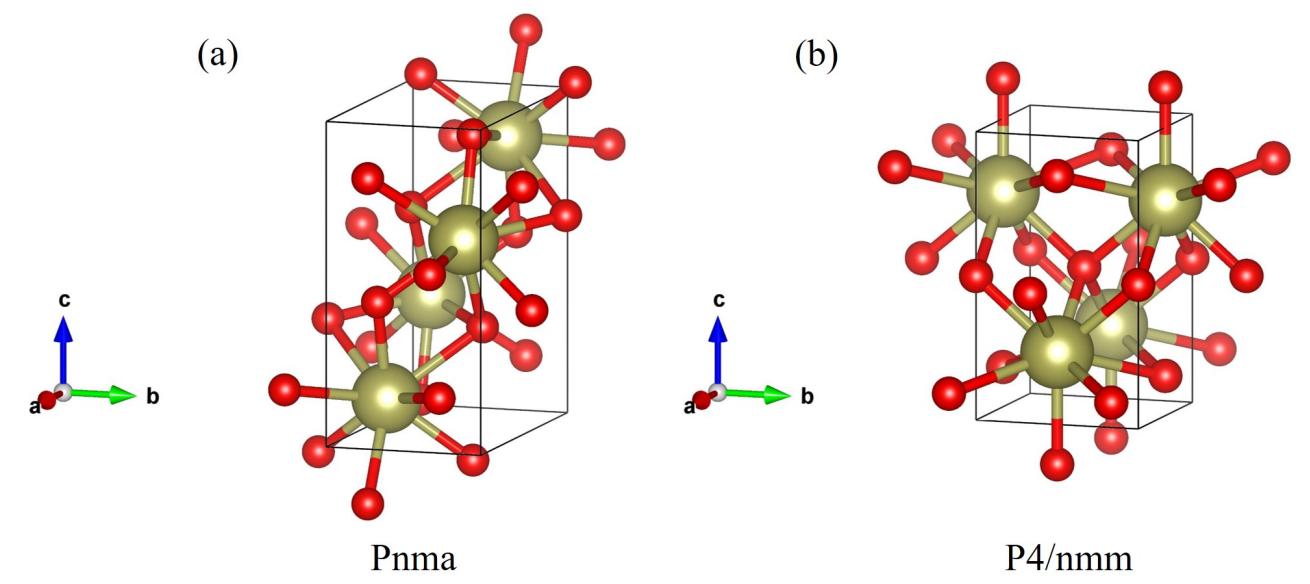

$\mathrm{P} 4 / \mathrm{nmm}$

Figure 1: Atomic configurations of the predicted $\mathrm{HfO}_{2}$ phase (a) the Pnma structure and (b) the $\mathrm{P} 4 / \mathrm{nmm}$ structure. Gold and red spheres, respectively, represent $\mathrm{Hf}$ and $\mathrm{O}$ atoms.

Regarding the dynamically stable structure, we have completed the phonon calculations by considering the $\mathrm{P} 4 / \mathrm{nmm}$ structure. According to the aforementioned relative enthalpy findings, one can see that the $\mathrm{P} 4 / \mathrm{nmm}$ structure is thermodynamically stable favored over the Pnma structure at a pressure 


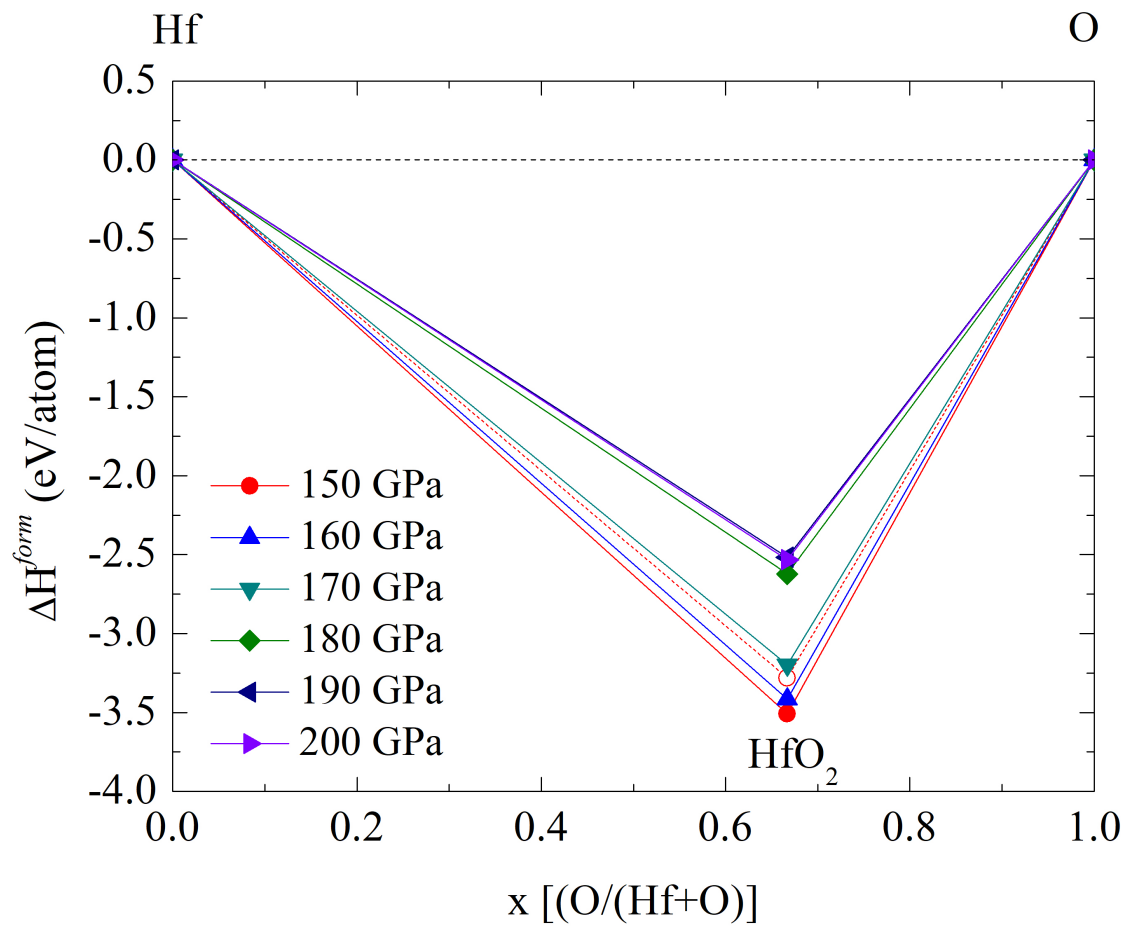

Figure 2: Formation enthalpy of $\mathrm{HfO}_{2}$ as a function of pressure, as calculated with respect to hafnium and oxygen, ranging from $150 \mathrm{GPa}$ to $200 \mathrm{GPa}$.

of $150 \mathrm{GPa}$. At this point, it is worth to investigate the dynamically stability of the $\mathrm{P} 4 / \mathrm{nmm}$ structure. The remarkable results, we found that the $\mathrm{P} 4 / \mathrm{nmm}$ structure is dynamically stable at a pressure of $150 \mathrm{GPa}$ because it lack an imaginary frequency, as seen in Figure3a. With increasing pressure, the P4/nmm structure is still dynamically stable at a pressure of $200 \mathrm{GPa}$. (Figure3b) It is interesting to note that the optical frequencies are significantly higher than that at a pressure of $150 \mathrm{GPa}$. As a possible cause of this, one might think of the effect of pressure. As a result of this, the P4/nmm structure will be investigated the electronic and mechanical properties, and is going to be further discussed in the following paragraphs.

To inspect the physical property of the $\mathrm{P} 4 / \mathrm{nmm}$ structure, we considered its density of states (DOS) at each pressure The results of the DOS are presented in Figure3, where those of $\mathrm{Hf}$ and $\mathrm{O}$ atoms are also shown for red and dark blue lines, respectively, as well as the total of DOS represents a dot 

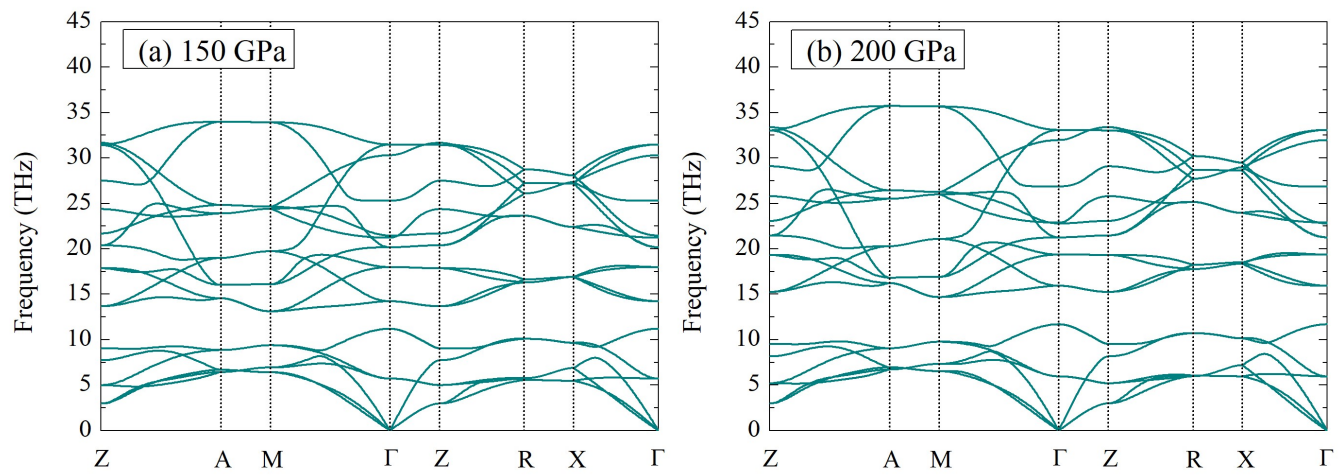

Figure 3: The phonon dispersions of the tetragonal structure (a) at a pressure of $150 \mathrm{GPa}$ and (b) at a pressure of $200 \mathrm{GPa}$.

line. As a result of this, the $\mathrm{P} 4 / \mathrm{nmm}$ structure is a semiconductor, moreover, the DOS displayed the band gap semiconductor with the band gap of $0.77 \mathrm{eV}$ at a pressure of $150 \mathrm{GPa}$. With increasing pressure, the DOS of the P4/nmm structure exhibited the band gap of the $0.86 \mathrm{eV}$, in addition, we computed the DOS at a pressure of $180 \mathrm{GPa}$ and found that the P4/nmm structure shown the band gap semiconductor with the band gap of $0.86 \mathrm{eV}$. Interestingly, the band gap semiconductor of the $\mathrm{P} 4 / \mathrm{nmm}$ structure decreased with increasing pressure of $200 \mathrm{GPa}$, resulting in the estimated the band gap semiconductor with the band gap of $0.15 \mathrm{eV}$. An observable effect of pressure in the P4/nmm structure is likely to be metallicity. Following this, it should note here that the more significant change in the shape of DOS is observed when the pressure increased. These findings mean that it was first investigated by effect of this electronic topological transition (ETT), indicating that the energy gap was falling down monotonically.

As mentioned above, the $\mathrm{P} 4 / \mathrm{nmm}$ structure it is the dynamically stable phases in the absence of imaginary frequency, and it is interesting to demonstrate the mechanical property. At this stage, the $\mathrm{P} 4 / \mathrm{nmm}$ structure mainly discussed in an elastic calculation. Here, we demonstrated an elastic stability conditions in the $\mathrm{P} 4 / \mathrm{nmm}$ structure, by considering the simplest form of elastic matrix, thus have 6 independent elastic constants [26], which defined as 

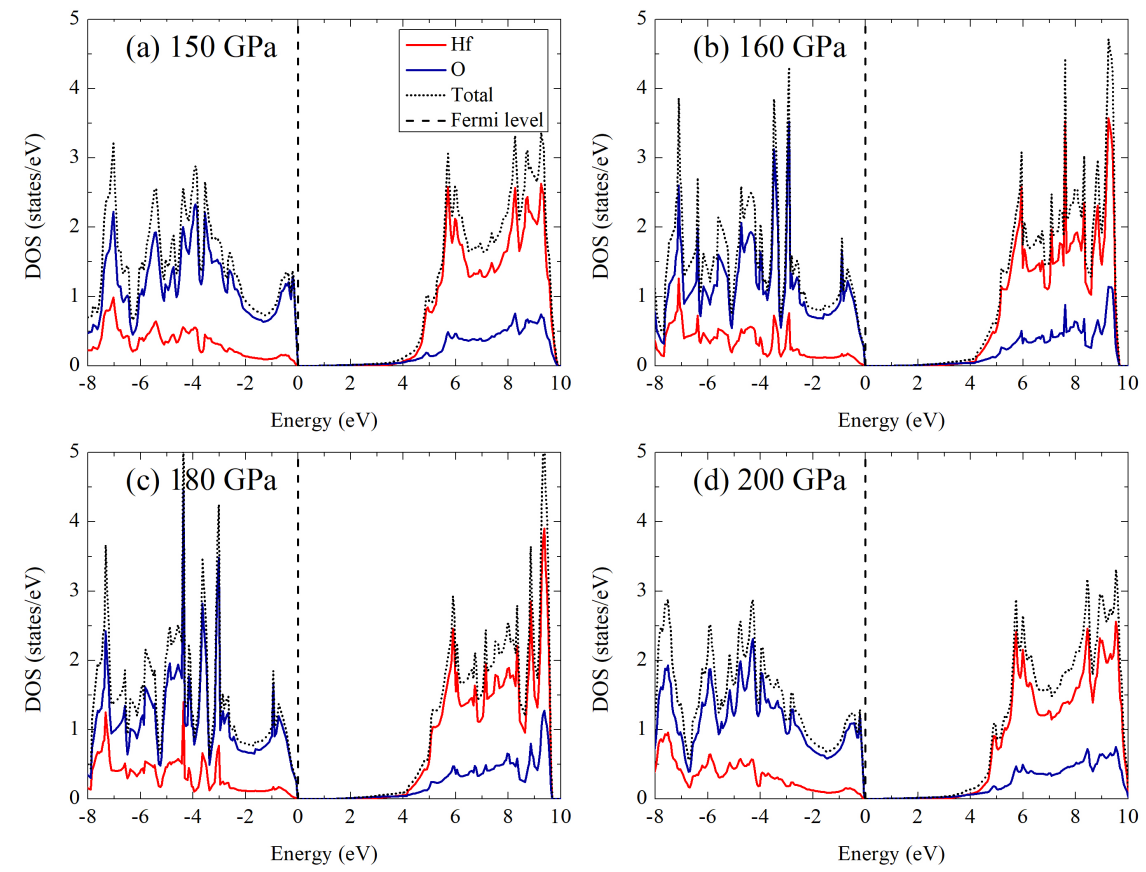

Figure 4: The density of states of the tetragonal structure is calculated at each pressure: (a) $150 \mathrm{GPa}$, (b) $160 \mathrm{GPa}$, (c) $180 \mathrm{GPa}$, and (d) $200 \mathrm{GPa}$.

$$
C_{i j}=\left(\begin{array}{cccccc}
C_{11} & C_{12} & C_{13} & & & \\
\cdot & C_{11} & C_{13} & & & \\
\cdot & \cdot & C_{33} & & & \\
& & & C_{44} & & \\
& & & & C_{44} & \\
& & & & & C_{66}
\end{array}\right)
$$

To further understand the mechanical stability criteria, it is theoretically calculated with the following these equations;

$$
\begin{gathered}
C_{11}>0, C_{33}>0, C_{44}>0, C_{66}>0 \\
\left(C_{11}-C_{12}\right)>0,\left(C_{11}+C_{33}-2 C_{13}\right)>0,
\end{gathered}
$$


Table 2: Elastic constants as a function of the $\mathrm{HfO}_{2}$

\begin{tabular}{lllllll}
\hline Pressure (GPa) & $\mathrm{C}_{11}$ & $\mathrm{C}_{12}$ & $\mathrm{C}_{13}$ & $\mathrm{C}_{33}$ & $\mathrm{C}_{44}$ & $\mathrm{C}_{66}$ \\
\hline 150 & 1149 & 606 & 543 & 1487 & 453 & 318 \\
160 & 1172 & 629 & 557 & 1519 & 469 & 322 \\
170 & 1199 & 656 & 572 & 1556 & 486 & 326 \\
180 & 1225 & 683 & 587 & 1593 & 504 & 329 \\
190 & 1251 & 710 & 602 & 1628 & 522 & 332 \\
200 & 1277 & 737 & 621 & 1675 & 539 & 333 \\
\hline
\end{tabular}

$$
\left[2\left(C_{11}+C_{12}\right)+C_{33}+4 C_{13}\right]>0 .
$$

As a result of elastic constants, we found that 6 independent elastic constants displayed all eigenvalues of $C_{i j}$ are positive, as shown in the Table- 2 . Also, the P4/nmm structure is corresponded with two criteria: (i) its phonon modes exhibited positive frequencies for all wave vectors and (ii) the elastic energy displayed always positive. The condition is well-known the elastic stability criterion. By analyzing the elastic property of the $\mathrm{P} 4 / \mathrm{nmm}$ structure, we considered the elastic constants as function of pressure. Fallowing, the results shown that the elastic constants increased with increasing pressure, in addition, it should be mentioned that the $C_{33}$ turned out to be large and it increased gradually from $150 \mathrm{GPa}$ to $200 \mathrm{GPa}$. Subsequently, it is possible that $C_{33}$ is a key factor for support the hardness property.

As mentioned hardness property, as first step, we investigated the bulk modulus (B), shear modulus $(\mathrm{G})$, and Young's modulus (E) of the P4/nmm structure by using the relation of elastic constants and calculating the VRH approximation. [27] Secondly, we used the Chen model to determine the Vicker harness as described in Ref. [6]. That is,

$$
H_{v}=2\left(k^{2} G\right)^{0.585}-3
$$

This model is described by the empirical formula, based on the Pugh modulus ratio $k=\mathrm{G} / \mathrm{B}$. We computed the Vicker's hardness as a function of pressure. As a result of this, it found that the Vicker's hardness increased significantly from $150 \mathrm{GPa}$ to $200 \mathrm{GPa}$. These findings tempt one to think of the $C_{33}$ because this correspondingly results in similarity in the trend of the Vicker's hardness as a function of pressure. We therefore suggested that this evidently confirms the increased Vicker's hardness.

To further understand the hardness properties, we described an electron 
Table 3: Calculated Bulk Modulus (B), Shear Modulus (G), Young's Modulus (E), Pugh modulus ratio $k=\mathrm{G} / \mathrm{B}$, and Vickers Hardness (Hv), respectively, of the $\mathrm{HfO}_{2}$

\begin{tabular}{llllll}
\hline Pressure $(\mathrm{GPa})$ & $\mathrm{B}$ & $\mathrm{G}$ & $\mathrm{E}$ & $\mathrm{G} / \mathrm{B}$ & $\mathrm{H}_{v}$ \\
\hline 150 & 796 & 384 & 994 & 0.482 & 24.7 \\
160 & 816 & 393 & 1017 & 0.481 & 25.0 \\
170 & 839 & 403 & 1043 & 0.480 & 25.3 \\
180 & 862 & 413 & 1069 & 0.479 & 25.7 \\
190 & 884 & 423 & 1094 & 0.478 & 26.0 \\
200 & 910 & 432 & 1120 & 0.475 & 26.1 \\
\hline
\end{tabular}
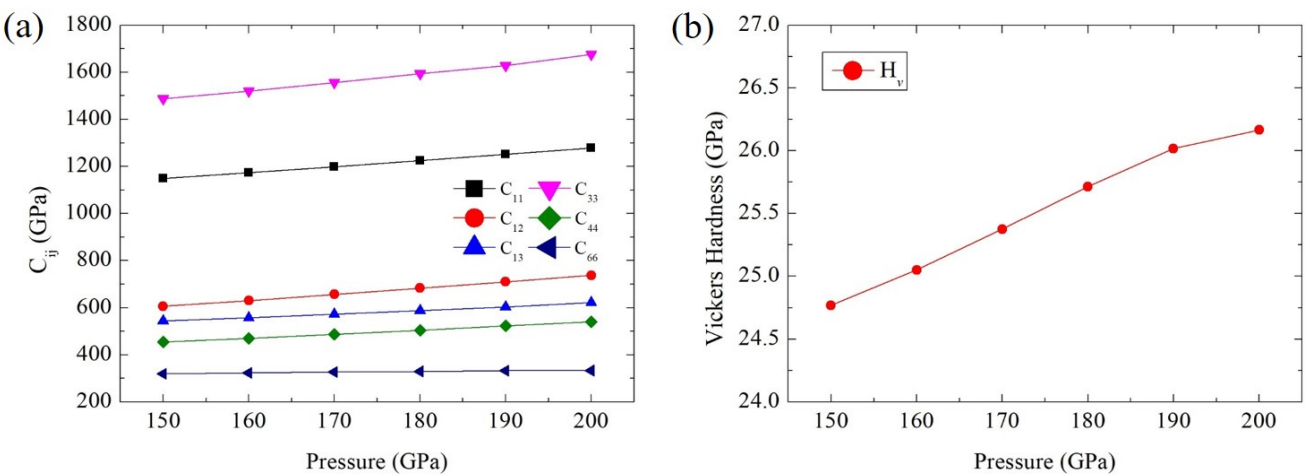

Figure 5: (a) Elastic constants of tetragonal structure as a function of pressure and (b) Vicker hardness of as a function of pressure.

in the neighboring space of $\mathrm{HfO}_{2}$ can be obtained by the electron localization function (ELF) [28], as reported in the (101) plane, where the structure of $\mathrm{HfO}_{2}$ is presented as shown in Figure 6. For the P4/nmm structure, the calculated ELF reveals a set of chemical bonding at the pressure of $150 \mathrm{GPa}$, by adopting the uniform distribution of electron gas with the same density $[17,18,29,30,31,32,33]$. The distances between the first (Hf-O) and second $(\mathrm{O}-\mathrm{O})$ nearest neighbors $(\mathrm{NN})$ read $1.937 \AA$, and $2.347 \AA$, respectively. As a result of the $\mathrm{P} 4 / \mathrm{nmm}$ structure, it can be observed that the electron would accumulate around the $\mathrm{Hf}$ and $\mathrm{O}$ atoms, respectively. Also, with increasing pressure up to $200 \mathrm{GPa}$, it is found that the distances between the first (Hf-O) and second (O-O) NN read $1.912 \AA$, and $2.307 \AA$, respectively. It should be noted, moreover, that the $\mathrm{O}$ atoms are likely to very weak bond. Nevertheless, it is worth noting that electrons at a pressure of $200 \mathrm{GPa}$ are 
slightly accumulated higher than at a pressure of $150 \mathrm{GPa}$, as can be seen in red circle dot (Figure 6). As a result of this, one might think that the nature of electrons accumulated the first (Hf-O) NN supports the increased Vicker's hardness.

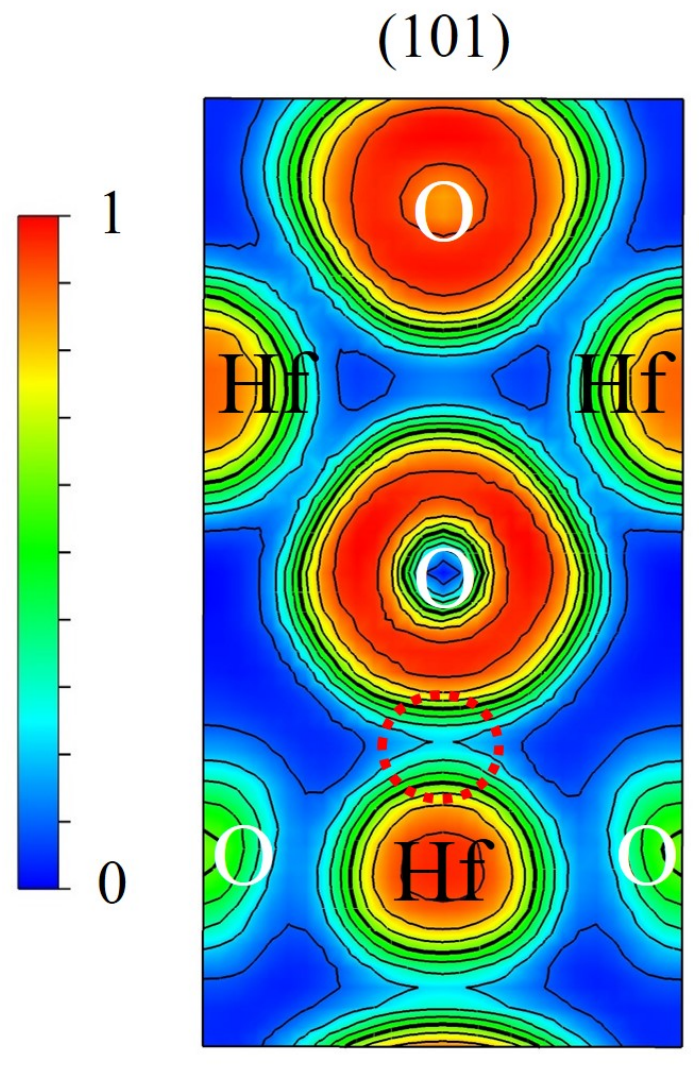

(a) $150 \mathrm{GPa}$

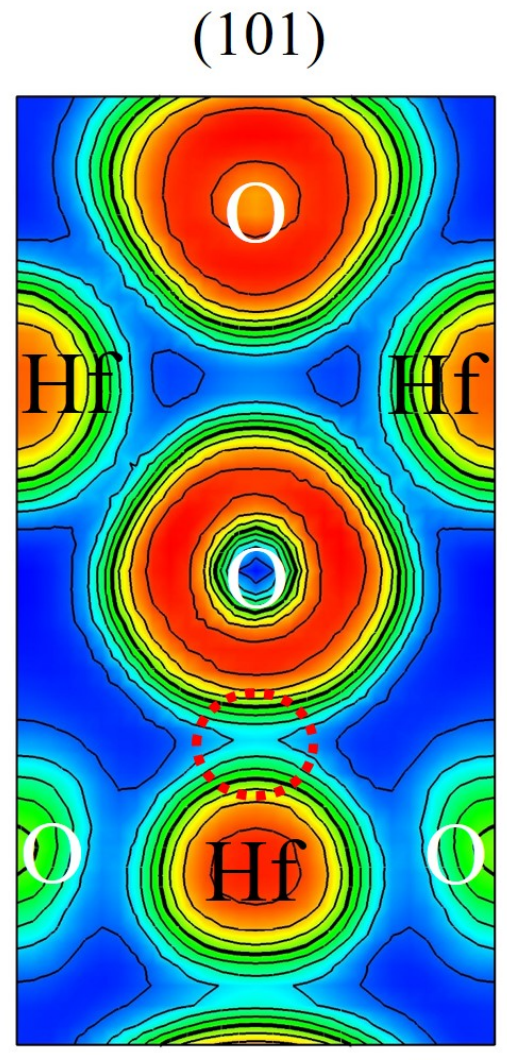

(b) $200 \mathrm{GPa}$

Figure 6: The electron localization function (ELF) in $\mathrm{HfO}_{2}$ : (a) ELF of the P4/nmm structure in the (101) atomic plane at $150 \mathrm{GPa}$ and (b) ELF of the P4/nmm structure in the (101) atomic plane at $200 \mathrm{GPa}$ (drawn by VESTA (ver. 3.4.7) [34] (URL https://jpminerals.org/vesta/en/download.html)).

\section{Conclusion}

In summary, we have performed the first-principles evolutionary techniques to predict an allotrope of $\mathrm{HfO}_{2}$ at high pressure. Regarding the 
structural predictions, we find an orthrhombic structure and a tetragonal structure with the space group of Pnma and the space group of $\mathrm{P} 4 / \mathrm{nmm}$. The remarkable result of the relative enthalpy shows that the $\mathrm{P} 4 / \mathrm{nmm}$ structure is thermodynamically favored over the Pnma structure at a pressure of $150 \mathrm{GPa}$. Apart from this, the $\mathrm{P} 4 / \mathrm{nmm}$ structure is dynamically stable. This evidently is conformed by the phonon calculations. Along with, the density of states suggests that hafnium dioxide allotrope is semiconductor. Following this, it finds that the energy gap is falling down monotonically, by adopting an effect of this electronic topological transition, resulting in the more significant change in the shape of DOS is observed when the pressure increased. By inspecting its Vicker's harness, the $\mathrm{P} 4 / \mathrm{nmm}$ structure displayed the Vicker's harness of $26.1 \mathrm{GPa}$ at a pressure of $200 \mathrm{GPa}$.

\section{5. acknowledgement}

We gratefully acknowledge the NSC (National Computer Center, Linköping, Sweden) in Sweden for providing computing time. This research project is supported by the Second Century Fund (C2F), Chulalongkorn University. R.A. thanks the Swedish Research Council (VR-2016-06014 \& VR2020-04410) for financial support. T.B. acknowledges the Development and Promotion of Science and Technology Talents Project (DPST).

\section{References}

[1] J. Haines, J. Léger, G. Bocquillon, Synthesis and design of superhard materials, Annual Review of Materials Research 31 (1) (2001) 1-23. arXiv:https://doi.org/10.1146/annurev.matsci.31.1.1, doi:10.1146/annurev.matsci.31.1.1.

URL https://doi.org/10.1146/annurev.matsci.31.1.1

[2] R. Andrievski, Superhard materials based on nanostructured highmelting point compounds: achievements and perspectives, International Journal of Refractory Metals and Hard Materials 19 (4) (2001) 447-452, sCIENCE OF HARD MATERIALS-7. SELECTED PAPERS FROM THE 7TH INTERNATIONAL CONFERENCE ON THE SCIENCE OF HARD MATERIALS. 
[3] V. V. Brazhkin, A. G. Lyapin, R. J. Hemley, Harder than diamond: Dreams and reality, Philosophical Magazine A 82 (2) (2002) 231-253. arXiv:https://doi.org/10.1080/01418610208239596, doi: $10.1080 / 01418610208239596$.

URL https://doi.org/10.1080/01418610208239596

[4] F. Gao, J. He, E. Wu, S. Liu, D. Yu, D. Li, S. Zhang, Y. Tian, Hardness of covalent crystals, Phys. Rev. Lett. 91 (2003) 015502. doi:10.1103/PhysRevLett.91.015502.

URL https://link.aps.org/doi/10.1103/PhysRevLett.91.015502

[5] H. Katzke, U. Bismayer, P. Tolédano, Theory of the high-pressure structural phase transitions in si, ge, sn, and pb, Phys. Rev. B 73 (2006) 134105. doi:10.1103/PhysRevB.73.134105.

URL https://link.aps.org/doi/10.1103/PhysRevB.73.134105

[6] X.-Q. Chen, H. Niu, D. Li, Y. Li, Modeling hardness of polycrystalline materials and bulk metallic glasses, Intermetallics 19 (9) (2011) 12751281.

[7] J. T. Mazumder, R. Mayengbam, S. Tripathy, Theoretical investigation on structural, electronic, optical and elastic properties of $\mathrm{TiO}_{2}, \mathrm{SnO}_{2}$, $\mathrm{ZrO}_{2}$ and $\mathrm{HfO}_{2}$ using $S C A N$ meta- $G G A$ functional: A DFT study, Materials Chemistry and Physics 254 (2020) 123474.

[8] S. Desgreniers, K. Lagarec, High-density $\mathrm{ZrO}_{2}$ and $\mathrm{H} \mathrm{fO}_{2}$ : crystalline structures and equations of state, Phys. Rev. B 59 (1999) 8467-8472.

[9] J. Zhang, A. R. Oganov, X. Li, K.-H. Xue, Z. Wang, H. Dong, Pressureinduced novel compounds in the $H f-O$ system from first-principles calculations, Phys. Rev. B 92 (2015) 184104.

[10] M. Berdova, X. Liu, C. Wiemer, A. Lamperti, G. Tallarida, E. Cianci, M. Fanciulli, S. Franssila, Hardness, elastic modulus, and wear resistance of hafnium oxide-based films grown by atomic layer deposition, Journal of Vacuum Science \& Technology A 34 (5) (2016) 051510. arXiv:https://doi.org/10.1116/1.4961113, doi:10.1116/1.4961113.

URL https://doi.org/10.1116/1.4961113 
[11] A. R. Oganov, C. W. Glass, Crystal structure prediction using ab initio evolutionary techniques: Principles and applications, The Journal of Chemical Physics 124 (24) (2006) 244704.

[12] G. Kresse, J. Furthmüller, Efficient iterative schemes for ab initio totalenergy calculations using a plane-wave basis set, Phys. Rev. B 54 (1996) 11169-11186.

[13] J. P. Perdew, K. Burke, M. Ernzerhof, Generalized gradient approximation made simple, Phys. Rev. Lett. 77 (1996) 3865-3868.

[14] P. E. Blöchl, Projector augmented-wave method, Phys. Rev. B 50 (1994) 17953-17979.

[15] A. Togo, I. Tanaka, First principles phonon calculations in materials science, Scr. Mater. 108 (2015) 1-5.

[16] P. Tsuppayakorn-aek, W. Luo, R. Ahuja, T. Bovornratanaraks, The high-pressure superconducting phase of arsenic, Scientific Reports 8 (1) (2018) 3026.

[17] P. Tsuppayakorn-aek, W. Luo, T. Watcharatharapong, R. Ahuja, T. Bovornratanaraks, Structural prediction of host-guest structure in lithium at high pressure, Scientific Reports 8 (1) (2018) 5278.

[18] P. Tsuppayakorn-aek, W. Luo, W. Pungtrakoon, K. Chuenkingkeaw, T. Kaewmaraya, R. Ahuja, T. Bovornratanaraks, The ideal commensurate value of $S c$ and the superconducting phase under high pressure, Journal of Applied Physics 124 (22) (2018) 225901.

[19] P. Jimlim, P. Tsuppayakorn-aek, T. Pakornchote, A. Ektarawong, U. Pinsook, T. Bovornratanaraks, Theoretical predictions for lowtemperature phases, softening of phonons and elastic stiffnesses, and electronic properties of sodium peroxide under high pressure, RSC Adv. 9 (2019) 30964-30975.

[20] P. Pluengphon, T. Bovornratanaraks, S. Vannarat, U. Pinsook, Structural and mechanical properties of GaAs under pressure up to 200 gpa, Solid state communications 195 (2014) 26-30. 
[21] P. Tsuppayakorn-aek, W. Chaimayo, U. Pinsook, T. Bovornratanaraks, Existence of the $\beta$-tin structure in sr: First evidence from computational approach, AIP Advances 5 (9) (2015) 097202. arXiv:https://doi.org/10.1063/1.4931810.

[22] K. Kotmool, P. Tsuppayakorn-aek, T. Kaewmaraya, U. Pinsook, R. Ahuja, T. Bovornratanaraks, Structural phase transitions, electronic properties, and hardness of $R u B_{4}$ under high pressure in comparison with $\mathrm{FeB}_{4}$ and $\mathrm{OsB}_{4}$, The Journal of Physical Chemistry C (06 2020).

[23] P. Tsuppayakorn-aek, J. Zhang, W. Luo, Y. Ding, R. Ahuja, T. Bovornratanaraks, Bain deformation mechanism and lifshitz transition in magnesium under high pressure, physica status solidi (b) 258 (3) (2021) 2000279 .

[24] H. Xia, G. Parthasarathy, H. Luo, Y. K. Vohra, A. L. Ruoff, Crystal structures of group IV metals at ultrahigh pressures, Phys. Rev. B 42 (1990) 6736-6738.

[25] Y. Ma, A. R. Oganov, C. W. Glass, Structure of the metallic $\zeta$-phase of oxygen and isosymmetric nature of the $\epsilon-\zeta$ phase transition: Ab initio simulations, Phys. Rev. B 76 (2007) 064101.

[26] F. Mouhat, F. m. c.-X. Coudert, Necessary and sufficient elastic stability conditions in various crystal systems, Phys. Rev. B 90 (2014) 224104. doi:10.1103/PhysRevB.90.224104.

URL https://link.aps.org/doi/10.1103/PhysRevB.90.224104

[27] D. H. Chung, W. R. Buessem, The voigt-reuss-hill (vrh) approximation and the elastic moduli of polycrystalline zno, tio2 (rutile), and -al2o3, Journal of Applied Physics 39 (6) (1968) 2777-2782.

[28] A. D. Becke, K. E. Edgecombe, A simple measure of electron localization in atomic and molecular systems, The Journal of Chemical Physics 92 (9) (1990) 5397-5403.

[29] T. Bovornratanaraks, P. Tsuppayakorn-aek, W. Luo, R. Ahuja, Groundstate structure of semiconducting and superconducting phases in xenon carbides at high pressure, Scientific Reports 9 (1) (2019) 2459. 
[30] P. Tsuppayakorn-aek, U. Pinsook, W. Luo, R. Ahuja, T. Bovornratanaraks, Superconductivity of superhydride CeH10 under high pressure, Materials Research Express 7 (8) (2020) 086001.

[31] P. Tsuppayakorn-aek, X. Yang, P. Pluengphon, W. Luo, R. Ahuja, T. Bovornratanaraks, Route to high- $T_{c}$ superconductivity of $B C_{7}$ via strong bonding of boron-carbon compound at high pressure, Scientific reports 10 (1) (2020) 1-8.

[32] P. Tsuppayakorn-aek, W. Sukmas, R. Ahuja, W. Luo, T. Bovornratanaraks, Stabilization and electronic topological transition of hydrogenrich metal $\mathrm{Li}_{5} \mathrm{MoH}_{11}$ under high pressures from first-principles predictions, Scientific Reports 11 (1) (2021) 1-10.

[33] P. Tsuppayakorn-aek, P. Phansuke, P. Kaewtubtim, R. Ahuja, T. Bovornratanaraks, Enthalpy stabilization of superconductivity in an alloying $S-P-H$ system: First-principles cluster expansion study under high pressure, Computational Materials Science 190 (2021) 110282.

[34] K. Momma, F. Izumi, VESTA: a three-dimensional visualization system for electronic and structural analysis, Journal of Applied Crystallography 41 (3) (2008) 653-658. 